\title{
The photometric follow-up observations for transiting exoplanet $\mathrm{XO}-2 \mathrm{~b}$
}

\author{
Sheng-hong $\mathrm{Gu}^{1}$, Andrew Collier Cameron ${ }^{2}$, Xiao-bin Wang ${ }^{1}$, \\ Xiang-song Fang ${ }^{1}$, Dong-tao $\mathrm{Cao}^{1}$ and Li-yun Zhang $^{3}$ \\ ${ }^{1}$ National Astronomical Observatories/Yunnan Observatory, CAS, Kunming, China \\ email: shenghonggu@ynao.ac.cn \\ ${ }^{2}$ University of St. Andrews, Fife KY16 9SS, UK \\ ${ }^{3}$ Guizhou University, Guiyang, China
}

\begin{abstract}
Four new transit light curves of XO-2b obtained in 2008 and 2009, are analyzed by using MCMC algorithm, and the system parameters are derived. The result demonstrates that the orbital period of the system obtained from new observations is almost the same as Burke et al.'s one (2007), which does not confirm the result of Fernandez et al. (2009).
\end{abstract}

Keywords. planetary systems, eclipses, techniques: photometric

\section{Introduction}

The transiting hot Jupiter XO-2b was discovered by Burke et al. (2007), its radius, mass and orbital period are $0.98 \mathrm{R}_{J}, 0.57 \mathrm{M}_{J}$ and 2.615857 days, respectively, the host star XO-2 has high metallicity and high proper motion. Later, Fernandez et al. (2009) observed other six transit events of the system and derived precise radius $0.996 \mathrm{R}_{J}$ and mass $0.565 \mathrm{M}_{J}$ of the exoplanet. They also found that the orbital period of the system changed by $2.5 \sigma$. Thus, more observations for its transit events are needed to clarify whether the orbital period of the system is variable.

\section{Observations and data reduction}

The new observations for transit events of XO-2b were made by using $85 \mathrm{~cm}$ telescope with $1 \mathrm{~K} x 1 \mathrm{~K}$ CCD camera (Zhou et al. 2009) of Xinglong station, NAOC on Dec.3, 2008 and $1 \mathrm{~m}$ telescope with $1 \mathrm{Kx} 1 \mathrm{~K}, 2 \mathrm{Kx} 2 \mathrm{~K}$ CCD cameras of Yunnan Observatory on Jan.19, 2008, Dec.7,12, 2009. In all observations, the R filter was employed. The observed CCD images are reduced by using IRAF package. For the obtained light curves, we remove the systematic errors by using coarse decorrelation method (Collier Cameron et al. 2006) and SysRem algorithm (Tamuz et al. 2005).

\section{Light curve analysis and discussion}

In order to get a set of precise system parameters for XO-2, the 4 datasets of transit events are combined in the course of light curve analysis. We model the flux of the transiting system with the parameters $\left\{\mathrm{T}_{c}, \mathrm{p}, \Delta \mathrm{F}, \mathrm{t}_{T}, \mathrm{~b}, \mathrm{M}_{*}\right\}$ considering the 4-coefficient limb-darkening law of Claret (2000). The basic parameters of the host star are adopted from the recent relative results (Fernandez et al. 2009). All observed data points are involved in MCMC (Markov Chain Monte Carlo) analysis to search the optimal parameters $\left\{\mathrm{T}_{c}, \mathrm{p}, \Delta \mathrm{F}, \mathrm{t}_{T}, \mathrm{~b}, \mathrm{M}_{*}\right\}$ according to the procedure of Collier Cameron et al. (2007). The 
Table 1. The optimal parameters derived for XO-2 system using the MCMC algorithm.

\begin{tabular}{ll}
\hline Transit epoch $\mathrm{T}_{c}$ [HJD] & $2455013.5983^{+0.0003}-0.0002$ \\
Orbital period $\mathrm{p}$ & $2.6158553^{+2.2 E-06}-2.5 E-06$ days \\
Transit depth $\Delta \mathrm{F}$ & $0.0136^{+0.0002}-0.0002$ mag. \\
Transit width $\mathrm{t}_{T}$ & $0.1094^{+0.0005}-0.0003$ days \\
Impact parameter b & $0.139^{+0.021}-0.070 \mathrm{R}_{*}$ \\
Orbital separation a & $0.0367915^{+1.5 E-06}-1.1 E-06 \mathrm{AU}$ \\
Orbital inclination i & $89.045^{+0.485}-0.147$ degrees \\
Stellar radius $\mathrm{R}_{*}$ & $0.951^{+0.001}-0.003 \mathrm{R}_{S u n}$ \\
Planet radius $\mathrm{R}_{p}$ & $0.945^{+0.006}-0.007 \mathrm{R}_{J}$ \\
Stellar mass $\mathrm{M}_{*}$ & $0.971 \mathrm{M}_{\text {Sun }}$ \\
Planet mass $\mathrm{M}_{p}$ & $0.565 \mathrm{M}_{J}$ \\
\hline
\end{tabular}
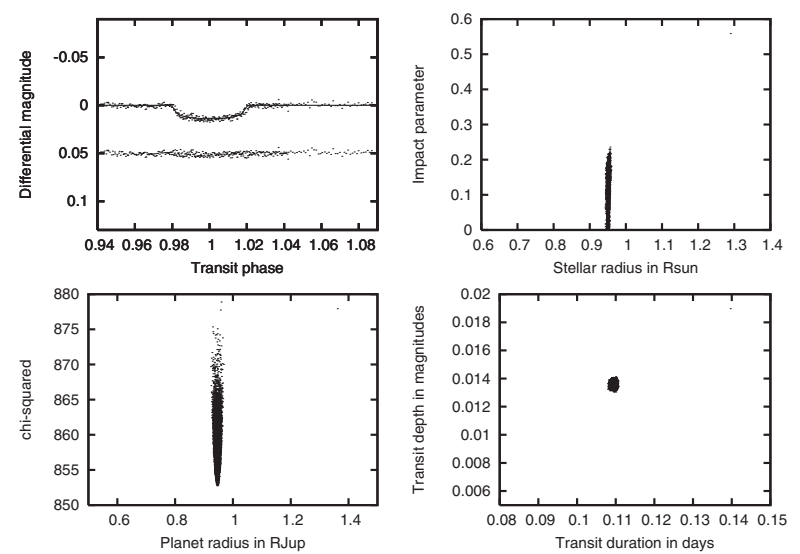

Figure 1. Binned light curve of $\mathrm{XO}-2 \mathrm{~b}$ and fitting information.

final result is listed in Table 1, the relative fitting and other information are shown in Fig. 1.

Compared our new result and the previous ones, it can be found that the new orbital period is almost the same as Burke et al.'s value (2.615857days). This does not support the suggestion that the orbital period of the system is probably variable, which was given by Fernandez et al. (2009). So, it is still necessary to observe more transit events for $\mathrm{XO}-2 \mathrm{~b}$ so as to make further investigation on its period behavior.

\section{Acknowledgments}

This work is supported by NSFC under grant No.10873031 and Chinese Academy of Sciences under grant KJCX2-YW-T24.

\section{References}

Burke, C. J., et al. 2007, ApJ, 671, 2115

Claret, A. 2000, A\&AA, 363, 1081

Collier Cameron, A., et al. 2006, MNRAS, 373, 799

Collier Cameron, A., et al. 2007, MNRAS, 380, 1230

Fernandez, J. M., et al. 2009, AJ, 137, 4911

Tamuz, O., Mazeh, T.,\& Zucker, S. 2005, MNRAS, 356, 1466

Zhou, A.-Y., et al. 2009, RAA, 9, 349 